\title{
Undulant Universe
}

\author{
Gabriela Barenboin* \\ Departament de Física Teòrica, Universitat de València, \\ Carrer Dr. Moliner 50, E-46100 Burjassot (València), Spain \\ Olga Mena Requejđ and Chris Quigd \\ Theoretical Physics Department, \\ Fermi National Accelerator Laboratory, \\ P.O. Box 500, Batavia, Illinois 60510 USA
}

(Dated: June 21, 2018)

\begin{abstract}
If the equation of state for "dark energy" varies periodically, the expansion of the Universe may have undergone alternating eras of acceleration and deceleration. We examine a specific form that survives existing observational tests, does not single out the present state of the Universe as exceptional, and suggests a future much like the matter-dominated past: a smooth expansion without a final inflationary epoch.
\end{abstract}

PACS numbers: 98.80.Cq, 95.35.+d, 98.70.Vc

FERMILAB-PUB-04-368-T

The discovery in measurements of distant supernova redshifts that the Universe is expanding at an accelerating pace 1, 2] has been reinforced and extended by detailed observations of anisotropies in the cosmic microwave background [3] and broad surveys of large-scale structure [4, 5]. The weight of observational evidence points to a flat Universe whose mass-energy includes $5 \%$ ordinary matter and $22 \%$ nonbaryonic dark matter, but is dominated by the "dark energy" identified as the motor for accelerated expansion. ${ }^{1}$ The inferred Universe has been variously described as extravagant [9] and preposterous [10] - extravagant, because we are acquainted with so little of the stuff that makes up the Universe, and preposterous because today's rough balance between matter and energy was unforeseen and might be a once in a lifetime occurrence, not just for observers, but also for the Universe!

Because the fossil record is spotty, and we are still learning how best to read it, there is much room for interpretation [11]. The most economical description of the cosmological measurements attributes the dark energy to a cosmological constant in Einstein's equation - an omnipresent and invariable vacuum energy density [12. A dynamical option is to suppose that a cosmic scalar field, called quintessence, changing with time and varying across space, is slowly approaching its ground state [13].

On the cosmological constant interpretation, the vacuum energy density would have made a scant contribution to the energy portfolio of the young small Universe, but the future Universe would grow so quickly as to be

*Gabriela.Barenboim@uv.es

†omena@fnal.gov

†quigg@fnal.gov

${ }^{1}$ For three complementary views of today's concordance cosmology, see Refs. $6,7,8]$. essentially empty of matter. Quintessence models admit many destinies. Neither proposal naturally explains why matter and vacuum energy should be of comparable importance at this moment in cosmic history. Responses to the "why now?" question range from anthropic rationalizations 14] to cyclic cosmologies [15].

In this Letter, we explore the possibility that the equation of state of the vacuum energy is an oscillatory function of the scale of the Universe. We shall show that a simple Ansatz compatible with existing observations responds naturally to the "why now?" problem and connotes a cosmic destiny similar to that of a matterdominated critical-density universe. In common with a cosmological constant, but in distinction to quintessence (which embodies a frozen field), the dynamical origin of such a universe could have been present at early times.

The expansion of the universe is determined by the Friedmann equation,

$$
H^{2} \equiv(\dot{R} / R)^{2}=8 \pi G_{\mathrm{N}} \rho / 3-k / R^{2}+\Lambda / 3
$$

where $H$ is the Hubble parameter, $R$ is the cosmological scale factor, $G_{\mathrm{N}}$ is Newton's constant, $\rho$ is the energy density, $k=(+1,0,-1)$ is the curvature constant, ${ }^{2}$ and $\Lambda$ is the cosmological constant. It is convenient to define the dimensionless scale factor, $a=R / R_{0}$, where the subscript 0 denotes the value at the current epoch. The critical density, defined from (11), is $\rho_{c}=3 H^{2} / 8 \pi G_{\mathrm{N}}$. The dimensionless cosmological density parameter is defined relative to the critical density as $\Omega_{\mathrm{tot}}=\rho / \rho_{c}$ at any epoch. We express the rate of change of the Hubble

\footnotetext{
${ }^{2}$ If $\Lambda=0$, the curvature constant determines destiny. For $k=+1$ (closed Universe), the Universe recollapses in finite time; for $k=$ 0 (flat) and $k=-1$ (open), the Universe expands without limit.
} 
parameter through the deceleration parameter,

$$
q \equiv-\frac{1}{H^{2}} \frac{\ddot{R}}{R}=\frac{\Lambda}{3 H^{2}}-\frac{4 \pi G_{\mathrm{N}}}{3 H^{2}}(\rho+3 p),
$$

where $p$ is the isotropic pressure. If we define $\Lambda=$ $4 \pi G_{\mathrm{N}} \rho_{\Lambda}$ and introduce the equation of state $w_{i}=p_{i} / \rho_{i}$ for any component of the universe, we can recast the deceleration parameter as

$$
q=\frac{1}{2} \sum_{i} \Omega_{i}\left(1+3 w_{i}\right)=\frac{1}{2}\left(\Omega_{\mathrm{tot}}+3 \sum_{i} \Omega_{i} w_{i}\right) .
$$

The equation of state of pressureless matter is $w_{m}=0$, and that of radiation is $w_{r}=\frac{1}{3}$. We see by inspection of Eq. (2) that $w_{\Lambda}=-1$.

The $\Lambda$ CDM proposal is parsimonious in its introduction of a single parameter, $\Omega_{\Lambda}$, but offers no explanation for the peculiar circumstance that $\Omega_{\Lambda} \approx \Omega_{m}$ at the current epoch - and no other - in the history of the universe. It is interesting to probe the range of interpretations that reproduce the observed features of the universe.

We investigate here the possibility that the physical characteristics of the vacuum energy vary with time, specifically with the number of $e$-foldings of the scale factor, with an equation of state

$$
w_{v}(a)=-\cos (\ln a)
$$

that matches the inference that $w_{v 0} \approx-1$ in the current universe. ${ }^{3}$ We assign the vacuum energy a weight $\Omega_{v 0}=$ 0.7 , in line with observations, and take $\Omega_{m 0}=0.3$ and $\Omega_{r 0}=4.63 \times 10^{-5}$. The present-day expansion rate is $H_{0}=100 h \mathrm{~km} \mathrm{~s}^{-1} \mathrm{Mpc}^{-1}$, with $h=0.71_{-0.03}^{+0.04}$ [17].

Because over one period the equation of state (4) averages to zero (the equation of state of pressureless matter), the cosmic coincidence problem is resolved. We plot in Figure 1 the normalized energy densities of matter, radiation, and vacuum energy as functions of the scale parameter $a$. These are given in terms of the normalized densities now as $\rho_{m} / \rho_{c 0}=\Omega_{m 0} / a^{3}, \rho_{r} / \rho_{c 0}=\Omega_{r 0} / a^{4}$, and $\rho_{v} / \rho_{c 0}=g(a) \Omega_{v 0} / a^{3}$, where

$$
g(a)=e^{3 \int_{a}^{1} d a^{\prime} w\left(a^{\prime}\right) / a^{\prime}}=e^{3 \sin (\ln a)} .
$$

Looking back in time to the epoch of big-bang nucleosynthesis at $a \approx 10^{-10}$, and forward to $a=10^{+10}$, we see that the vacuum energy density crosses the matter density every $\pi e$-foldings of the scale factor. These regular crossings stand in sharp contrast to the $\Lambda$ CDM cosmology, in which $\Lambda_{v} \approx \Lambda_{m}$ only in the current epoch. Periodically dominant dark energy is in the spirit of Refs. 18, 19].

\footnotetext{
3 Equations of state involving $\cos (\ln a)$ have been explored, to a different end, in Ref. [16].
}

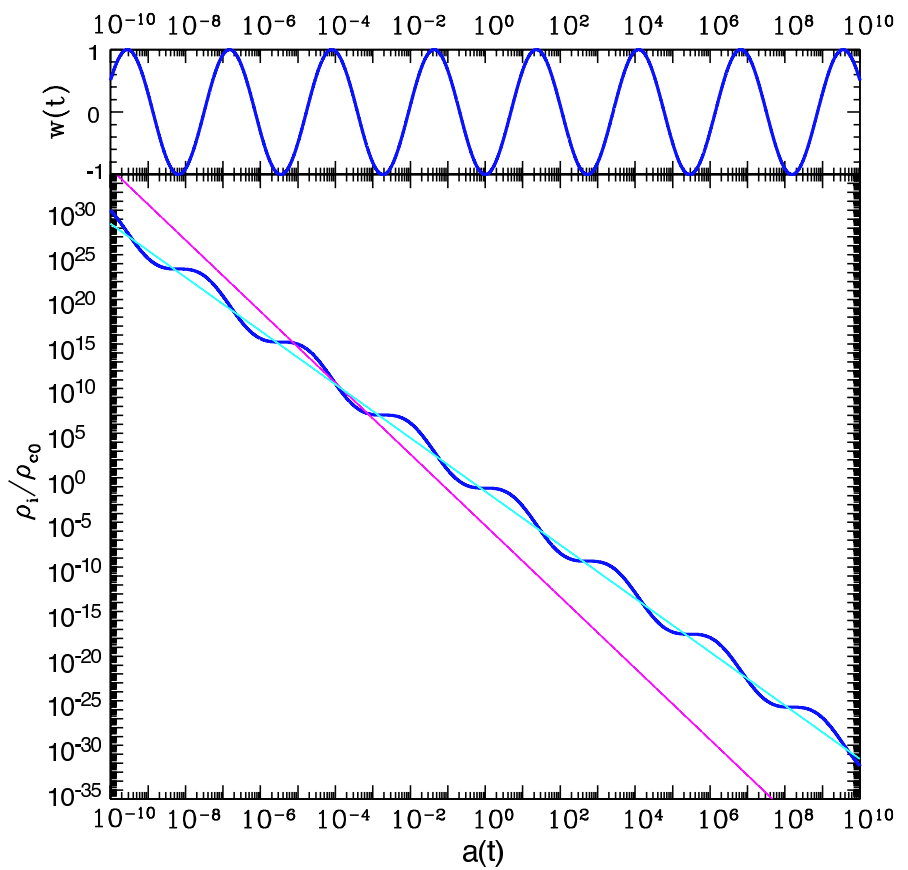

FIG. 1: Lower panel: Evolution of the matter (thin cyan), radiation (magenta, steepest line), and vacuum (thick blue) energy densities in the undulant universe, normalized to the critical density $\rho_{i} / \rho_{c 0}$, versus the scale factor $a(t)$. Upper panel: Equation of state, Eqn. (4), of the undulant vacuum.

The Hubble parameter is now given by

$$
H(a)=H_{0} \sqrt{\frac{\Omega_{m}}{a^{3}}+\frac{g(a) \Omega_{v}}{a^{3}}+\frac{\Omega_{r}}{a^{4}}},
$$

and the current age of the universe, $t_{0}=\int_{0}^{1} d a / H(a) a$, is 13.04 Gyr, to be compared with 13.46 Gyr in the $\Lambda$ CDM model. Both values are in good agreement with the age of $(12.9 \pm 2.9)$ Gyr inferred from globular clusters [20]. By calculating the time to reach a given scale factor, we can determine the history and future of the universe. During the radiation dominated era, which corresponds to $a \lesssim$ $10^{-5}, a(t) \propto t^{1 / 2}$; when matter dominates, $a(t) \propto t^{2 / 3}$.

We show the results for three cosmologies in Figure 2. The dashed (red) line corresponds to the "standard cold dark matter" (SCDM) cosmology that was canonical before the discovery of the accelerating universe. The thin solid (black) line shows the $\Lambda$ CDM cosmology, in which the present epoch marks the beginning of a final inflationary period that leads to an empty universe in which matter is a negligible component. The heavy (blue) line shows the prediction of Eqn. (4). In the recent past, the periodic equation of state matches the behavior of the $\Lambda \mathrm{CDM}$ cosmology, but in the future it undulates about the SCDM prediction.

The expansion of the undulant universe is characterized by alternating periods of acceleration and deceleration shown by the deceleration parameter in Figure 3. For scale factors $a$ between 0.1 and 1 , the periodic equa- 


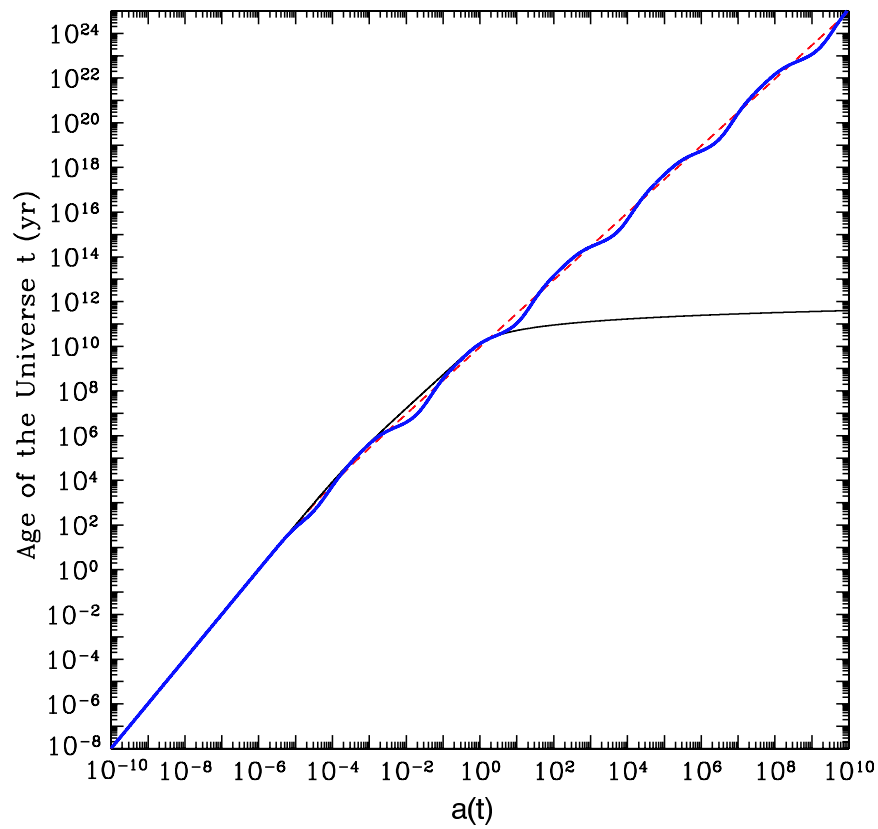

FIG. 2: Evolution of the scale factor $a(t)$ in three cosmologies: the canonical $\Lambda \mathrm{CDM}$ model (thin black line); a critical universe (SCDM model) with $\Omega_{m}=1$ (dashed red line); and the periodic equation of state (4) (thick blue line).

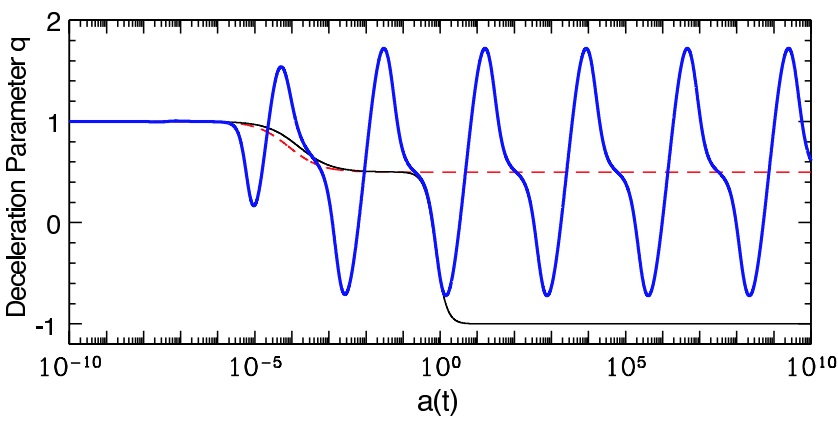

FIG. 3: The deceleration parameter as defined in Eq. (3) for the undulant universe (thick blue line), $\Lambda$ CDM model (thin black line) and SCDM model (dashed red line).

tion of state tracks the behavior of the $\Lambda$ CDM cosmology. But whereas $\Lambda \mathrm{CDM}$ is about to enter an era of sustained acceleration, the average behavior of the undulant universe tracks that of SCDM.

We have performed a number of checks to verify that the periodic equation of state is consistent with existing observations. At BBN, we find $\Omega_{v} \approx 2 \times 10^{-5}$, which respects the bound $\Omega_{v}<0.045$ (2 $\sigma$ limit) [21]. The model reproduces the luminosity distance modulus of the $\mathrm{Su}-$ pernova Search Team's gold and silver samples 22] and the $\mathrm{x}$-ray gas mass fractions determined by the Chandra X-ray Observatory [23, 24, 25]. We find no significant difference between the linear growth factors 26$]$ in the $\Lambda \mathrm{CDM}$ and undulant cosmologies. We will present details elsewhere.
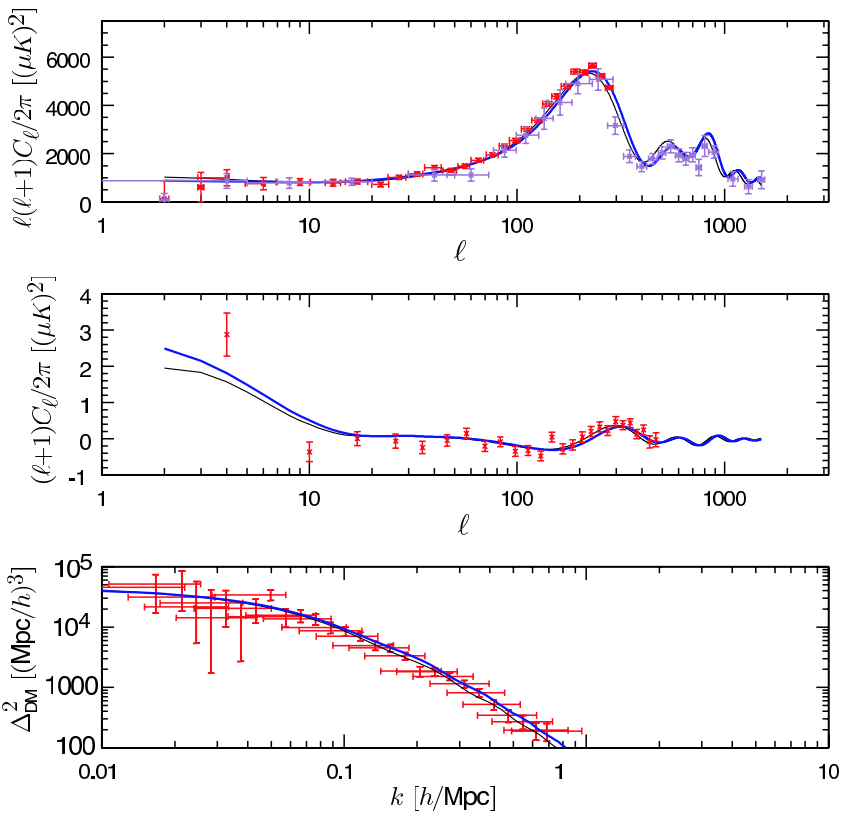

FIG. 4: Angular power spectrum (top panel) and T-E crosscorrelation (middle panel) versus the multipole $\ell$, and matter power spectrum versus the wave number $k$ (bottom panel), for the periodic equation of state (4) (blue line) and for the $\Lambda$ CDM model (black line). The top panel shows experimental data from the WMAP experiment (red) 28] and from the combination of all CMB data (purple) 29]. The middle panel shows WMAP data. The data in the bottom panel are from an independent analysis of the $2 \mathrm{dF}$ survey [30].

We have modified the CMBFAST 27] code to compute the implications of the periodic equation of state for the anisotropies of the cosmic microwave background. We show in Figure 4 that the undulant universe describes the angular power spectrum, temperature-polarization crosscorrelation, and matter power spectrum with the same degree of fidelity as the $\Lambda \mathrm{CDM}$ model.

The undulant universe offers a new response to the cosmic coincidence problem: the current state of the Universe, with $\Omega_{m} \approx \Omega_{v}$ and $w_{v} \approx-1$, has happened before and will happen again. No fine tuning is required, in the sense that $0.5 \leq \Omega_{v} \leq 0.9$ with $w_{v} \leq-0.7$ occurs with $\sim 10 \%$ probability for $10^{-10} \leq a \leq 10^{+10}$. We have not given a physical picture for the stuff that makes up the vacuum energy, though the program for constructing a potential in which a scalar field has the required behavior is clear [31, 32]. On the observational front, it is of clear importance to seek evidence that the vacuumenergy equation of state varies with time [33], and to find new constraints at different epochs.

Others have remarked 34] that no finite set of astronomical measurements made over a finite time will ever allow us to determine the ultimate fate of our Universe, and have quantified 35 the limited reach of reliable extrapolations. The undulant universe explored here shows that the range of possible destinies for the Universe, even 
in the near term, is very broad indeed. The universe need not necessarily evolve toward the cataclysm of terminal inflation or recollapse, but might continue the sedate drift of a big slink.

Fermilab is operated by Universities Research Association Inc. under Contract No. DE-AC02-76CH03000 with the U.S. Department of Energy. G.B. and O.M. acknowledge the stimulating environment of the Aspen Center for Physics. It is a pleasure to thank Sean Carroll, Scott Dodelson, Joe Lykken, Gary Steigman, Tim Tait, Jochen Weller, and Matias Zaldarriaga for enlightening conversations.

[1] A. G. Riess et al. [Supernova Search Team], Astron. J. 116, 1009 (1998) arXiv:astro-ph/9805201.

[2] S. Perlmutter et al. [Supernova Cosmology Project], Astrophys. J. 517, 565 (1999) arXiv:astro-ph/9812133.

[3] C. L. Bennett et al., Astrophys. J. Suppl. 148, 1 (2003) arXiv:astro-ph/0302207. A summary table of cosmological parameters is available at http://lambda.gsfc.nasa.gov/product/map/wmap_para

[4] The Two-Degree Field Galaxy Redshift Survey obtained spectra for 245591 objects, mainly galaxies; see http://www.mso.anu.edu.au/2dFGRS/

[5] The Sloan Digital Sky Survey, http://www.sdss.org measures distances to $\mathcal{O}\left(10^{6}\right)$ galaxies and quasars.

[6] P. J. E. Peebles and B. Ratra, Rev. Mod. Phys. 75, 559 (2003) arXiv:astro-ph/0207347.

[7] W. L. Freedman and M. S. Turner, Rev. Mod. Phys. 75, 1433 (2003) arXiv:astro-ph/0308418.

[8] M. Trodden and S. M. Carroll, "TASI lectures: Introduction to Cosmology," arXiv:astro-ph/0401547

[9] Robert P. Kirshner, The Extravagant Universe (Princeton University Press, Princeton, 2002).

[10] S. M. Carroll, "Dark Energy and the Preposterous Universe," arXiv:astro-ph/0107571 See also S. M. Carroll, "The Cosmological Constant," Living Rev. Relativity 4, 1 (2001), http://www.livingreviews .org/lrr-2001-1

[11] S. L. Bridle, O. Lahav, J. P. Ostriker and P. J. Steinhardt, Science 299, 1532 (2003) arXiv:astro-ph/0303180.

[12] L. M. Krauss and M. S. Turner, Gen. Rel. Grav. 27, 1137 (1995) arXiv:astro-ph/9504003.

[13] R. R. Caldwell, R. Dave and P. J. Steinhardt, Phys. Rev. Lett. 80, 1582 (1998) arXiv:astro-ph/9708069.

[14] L. Susskind, "The anthropic landscape of string theory," arXiv:hep-th/0302219

[15] P. J. Steinhardt and N. Turok, Science 296, 1436 (2002).

[16] B. Feng, M. Li, Y. S. Piao and X. Zhang,
"Oscillating quintom and the recurrent universe," arXiv:astro-ph/0407432

[17] S. Eidelman et al. [Particle Data Group], Phys. Lett. B 592, 1 (2004).

[18] S. Dodelson, M. Kaplinghat and E. Stewart, Phys. Rev. Lett. 85, 5276 (2000) arXiv:astro-ph/0002360.

[19] K. Griest, Phys. Rev. D 66, 123501 (2002) arXiv:astro-ph/0202052.

[20] J. Cepa, "Constraints on the cosmic equation of state: Age conflict versus phantom energy. Age-redshift relations in an accelerated universe," arXiv:astro-ph/0403616

[21] R. Bean, S. H. Hansen and A. Melchiorri, Nucl. Phys. Proc. Suppl. 110, 167 (2002) arXiv:astro-ph/0201127.

[22] A. G. Riess et al. [Supernova Search Team], Astrophys. J. 607, 665 (2004) arXiv:astro-ph/0402512 . For data analysis details see http://www.astro.ucla.edu/ wright/

[23] The Chandra X-Ray Observatory, http://chandra.nasa.gov

[24] S. W. Allen, R. W. Schmidt, H. Ebeling, A. C. Fabian and L. van Speybroeck, Mon. Not. Roy. Astron. Soc. 353, 457 (2004) arXiv:astro-ph/0405340; S. W. Allen, Private comunication.

[25] D. Rapetti, S. W. Allen and J. Weller, "Constraining Dark Energy with X-ray Galaxy Clusters, Su- fefm tovae and the Cosmic Microwave Background," arXiv:astro-ph/0409574

[26] E. V. Linder and A. Jenkins, Mon. Not. Roy. Astron. Soc. 346, 573 (2003) arXiv:astro-ph/0305286.

[27] U. Seljak and M. Zaldarriaga, Astrophys. J. 469, 437 (1996) arXiv:astro-ph/9603033; M. Zaldarriaga, private communication. The most recent version of the CMBFAST code is at http://www.cmbfast.org/

[28] WMAP power spectrum data can be found at http://lambda.gsfc.nasa.gov/product/map/

[29] X. Wang, M. Tegmark, B. Jain and M. Zaldarriaga, Phys. Rev. D 68, 123001 (2003) arXiv:astro-ph/0212417.

[30] M. Tegmark, A. J. S. Hamilton and Y. Xu, Mon. Not. Roy. Astron. Soc. 335, 887 (2002) arXiv:astro-ph/0111575.

[31] T. Nakamura and T. Chiba, Mon. Not. Roy. Astron. Soc. 306, 696 (1999) arXiv:astro-ph/9810447.

[32] B. F. Gerke and G. Efstathiou, Mon. Not. Roy. Astron. Soc. 335, 33 (2002) arXiv:astro-ph/0201336.

[33] U. Alam, V. Sahni, T. D. Saini and A. A. Starobinsky, arXiv:astro-ph/0311364

[34] L. M. Krauss and M. S. Turner, Gen. Rel. Grav. 31, 1453 (1999) arXiv:astro-ph/9904020

[35] Y. Wang, J. M. Kratochvil, A. Linde and M. Shmakova, "Current Observational Constraints on Cosmic Doomsday," arXiv:astro-ph/0409264 